
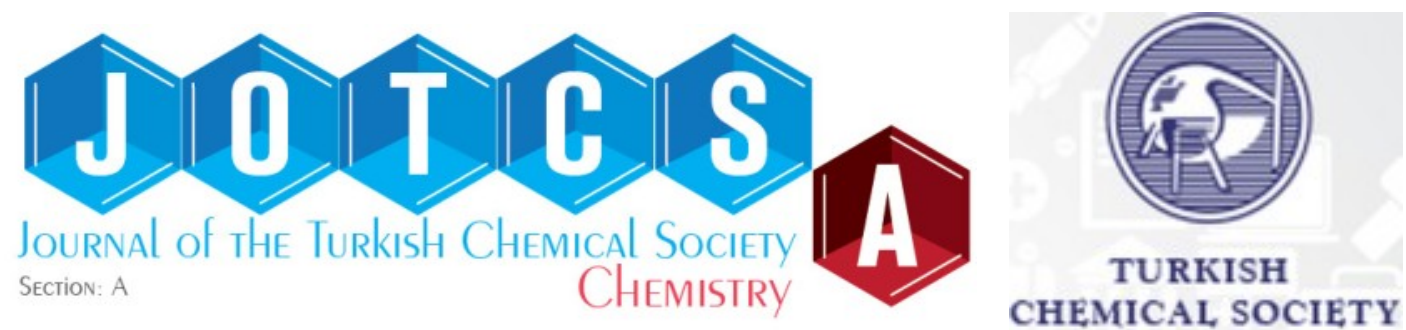

\title{
Effects of selected synthetic and biological insecticides on microbial population and microbial activities of soil samples
}

\author{
Sebiomo Adewole* $\triangle$ and Banjo Folake Mary $\square$ \\ Department of Biological Sciences, Tai Solarin University of Education, ljagun, Nigeria.
}

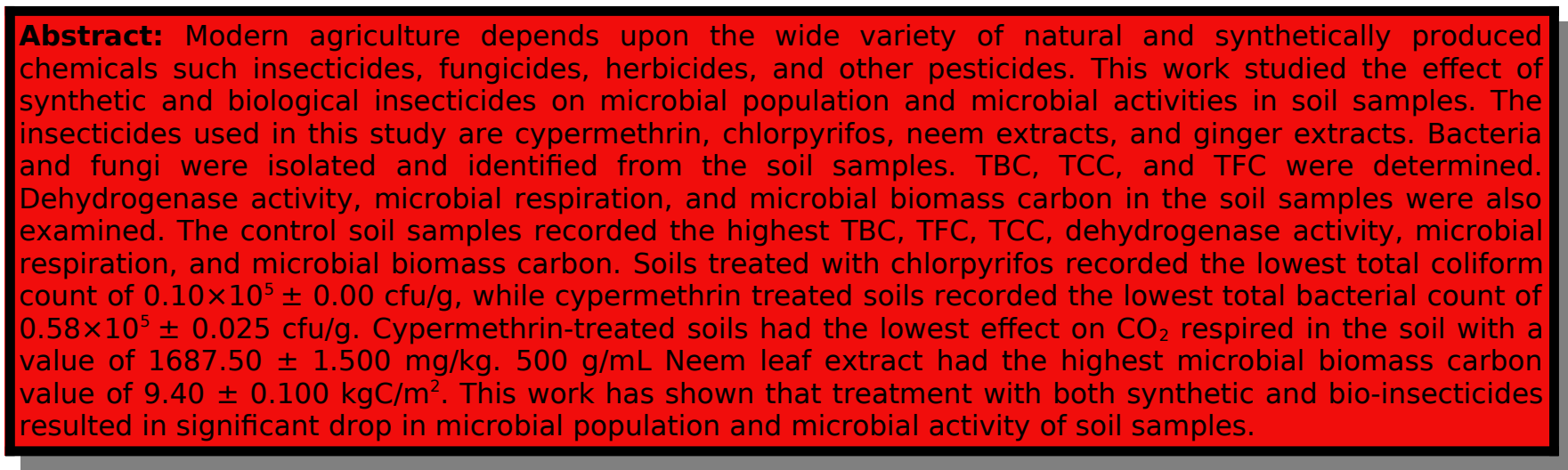

Keywords: Microbial population, dehydrogenase activity, microbial respiration, microbial biomass carbon, cypermethrin, chlorpyrifos.

Submitted: May 03, 2020. Accepted: December 27, 2020.

Cite this: Sebiomo A, Banjo F. Effects of selected synthetic and biological insecticides on microbial population and microbial activities of soil samples. JOTCSA. 2021;8(1):229-38.

DOI: https://doi.org/10.18596/jotcsa.731232.

*Corresponding author. E-mail: rev20032002@yahoo.com, Phone: +2348077675121 or +2347066468516 .

\section{INTRODUCTION}

Mechanized agriculture depends heavily upon synthetically manufactured chemicals such as insecticides, fungicides, herbicides, and other pesticides (1). When pesticides are introduced into the soil about, it reaches the target organisms, interferes with metabolism in the environment, affects physicochemical properties or enzymatic activities of the soil, and also invariably affects human health. This has resulted in considerable public concern (2). Microorganisms are important parts of the food web in soils. Microbial biomass is a measure of potential microbiological and ecosystem activities. In order to understand the functioning of the ecosystem and examine soil disturbance because of various agricultural management practices, microbial activities, and microbial biomass must be examined (3).

Insecticides are toxic substances which are used by farmers to kill insects which are liable to have a negative impact on crop production. Insecticides are used in agriculture, medicine, industry, and by consumers. They are an important factor behind the increase in agricultural productivity of the 20th century. Nearly all insecticides have the potential to significantly impact ecosystems; many are toxic to humans; while others accumulate in the environment (4). Residues of insecticides are broken down by a combination of environmental factors and microorganisms. Degradation products which are a result of microbial interactions leads to increased population sizes and microbial enzyme activities 
which can in turn affect the transformation of plant nutrient elements in soil (5). There are insecticides which can only be degraded in soil by microorganisms through cometabolism. Other insecticides exert the deleterious effect on microorganisms. The use of insecticides in crops protects plants against different groups of insect pests. Although these chemicals are applied in low concentrations once in the soil, they can alter the chemical and biological properties of it and also affect soil microorganisms.

Das and Mukherjee (5) reported stimulatory effects of carbofuran at different doses under laboratory condition (5). Insecticides are used on crop plants to protect them from different group of harmful insects. Application of insecticides results in the decrease of the number of micro-organisms, alteration in biochemical activities, and quantitative and qualitative decrease of the microbial community of soil samples (6). Large-scale use of common pesticides can lead to soil toxicity, which may disturb several bio-chemical reactions and soil physicochemical properties. Due to high degree of toxicity, some pesticides particularly those that are persistent in soils, constitute a very important group of contaminants. Application of insecticides to plants and invariably reaching soils usually leads to their interaction with non-target soil micro-organisms and physio-chemical properties and hence exhibit chronic diverse effects on soil microflora (7). Hence this work studied the effect of synthetic and biological insecticides on microbial population and microbial activities (dehydrogenase activity, microbial respiration, and microbial biomass carbon) of soil samples.

\section{MATERIALS AND METHODS}

\section{Soil sampling}

Soil sampling were done in triplicate (in completely randomized design), using hand trowel to collect samples from the research field of Biological Sciences Department, Tai-Solarin University of Education, ljagun, Ogun state, Nigeria. The samples were collected at $5 \mathrm{~cm}$ depth using the soil augur. The samples were then sieved with wire mesh (size $<2 \mathrm{~mm}$ ). Stones, plant debris and any visible soil fauna were removed from the soil samples by sorting after which they were thoroughly mixed with hand trowel. The soil was allowed to settle for seven days by incubating at $27{ }^{\circ} \mathrm{C}$ to allow the disturbances caused by sampling and sieving to stabilize. After the soil samples were allowed to settle, seeds of Celosia argentia were planted. Soil samples were collected 48 hours after treatment of Celosia argentia with the insecticides. The synthetic insecticides were applied according to manufacturer's instruction, in different plastic pots filled with $5 \mathrm{~kg}$ of soil and untreated soil samples were used as control. Neem and ginger extracts were also applied to different plastic pots filled with $5 \mathrm{~kg}$ soil samples. Soil samples were obtained from the pots before and after incubation, after which they were analyzed.

\section{Insecticides}

The insecticides which were used in this study were obtained from local stores in ljebu-Ode, Nigeria. The active ingredients are cypermenthrin $10 \%$ EC and chlorpyriphos 20\% EC. Biological insecticides such as neem leaf extract and ginger extracts were also used in this study.

\section{Preparation of plant extracts and insecticide sprays}

Fresh neem leaves and ginger was soaked with water for three days, then they were cut into pieces, crushed, and blended using an electric blending machine. Thereafter, the following increasing quantities were prepared $(100 \mathrm{~g} / \mathrm{mL}, 200 \mathrm{~g} / \mathrm{mL}, 300$ $\mathrm{g} / \mathrm{mL}, 400 \mathrm{~g} / \mathrm{mL}$, and $500 \mathrm{~g} / \mathrm{mL})$. After $24 \mathrm{~h}$, the extract solutions were sieved with a cheese cloth to obtain a clear appearance. Cypermethrin and chlorpyrifos were prepared according to the manufacturer's specified instructions. The Celosia argentia plants were then sprayed with equal quantities of the insecticides.

\section{Microbial analysis of soil samples}

Total heterotrophic bacteria in soil samples were analyzed using Nutrient Agar (NA). Incubation was done at $30{ }^{\circ} \mathrm{C}$ for $24-48 \mathrm{~h}$. Potato dextrose agar (PDA) was used for enumeration, isolation, and identification of fungi. Fungal isolates were incubated at $25{ }^{\circ} \mathrm{C}$ for 5-7 days. Bacterial isolates were characterized as based on cultural characteristics, staining reactions, and biochemical reactions and identifications were done using Bergey's Manual of Systemic Bacteriology (1984) as reference. Morphological and cultural characteristics were used to identify the fungi. The bacteria and fungi that emerged from plates were sub-cultured several times until pure cultures were obtained.

\section{Determination of dehydrogenase activity}

Six grams of soil and $6 \mathrm{~mL}$ of water samples were dispensed separately into $500 \mathrm{~mL}$ conical flasks. Thirty $\mathrm{mL}$ of glucose, $1 \mathrm{~mL}$ of 2,3,5triphenyltetrazolium chloride (TTC) solution plus 2.5 $\mathrm{mL}$ of distilled water were added and shaken for 5 min. The mixtures were then filtered through a double layered filter paper into a $250 \mathrm{~mL}$ conical flask having formed 1,3,5-triphenyl formazan (TPF). The absorbances of sample extracts were read on a Cecil UV/Vis Spectrophotometer at a wavelength of $485 \mathrm{~nm}(20)$

\section{Determination of microbial respiration}

The method of Klimek (8) was employed to determine soil microbial respiration. Soil samples were placed in glass jars containing $10 \mathrm{~mL}$ of $0.1 \mathrm{~N}$ $\mathrm{NaOH}$ solution. They were incubated in the dark at $25+0.5^{\circ} \mathrm{C}$. Soil moisture content was maintained at $60 \%$ water holding capacity. Soil $\mathrm{CO}_{2}$-evolution was regularly estimated during the twenty-five days incubation period and $\mathrm{CO}_{2}$ released was measured 
every 5 days. $\mathrm{CO}_{2}$ recovered in each $\mathrm{NaOH}$ solution was measured by titration with $\mathrm{HCl}$, after the addition of barium chloride. Percentage $\mathrm{CO}_{2}$ evolved was then calculated.

\section{Determination of microbial biomass carbon}

Microbial biomass carbon was determined using the method of Vance et al. (9). Insecticide treated soils $(5 \mathrm{~g})$ were fumigated with $50 \mathrm{~mL}$ of $2: 1$ chloroform- ethanol in a vacuum desiccator for $24 \mathrm{hrs}$. Soils that were not fumigated with chloroform-ethanol were used as control. The soil samples were extracted with $40 \mathrm{~mL}$ of $0.5 \mathrm{M} \mathrm{K}_{2} \mathrm{SO}_{4}$ for $30 \mathrm{~min}$ in an oscillator at $300 \mathrm{rpm}$. The control soil samples were also extracted with the $0.5 \mathrm{M} \mathrm{K}_{2} \mathrm{SO}_{4}$ and filtered through a Whatman No 42 Filter paper into a $250 \mathrm{~mL}$ conical flask. Microbial carbon was determined in a UV/Vis Spectrophotometer.

$$
\text { Microbial biomass carbon }=\frac{\text { Absorbance of sample } \times \text { Gradient Factor } \times \text { Dilution factor }}{\text { Weight of sample }}
$$

\section{Statistical analysis}

The data collected were subjected to analysis of variance (ANOVA). All analyses were carried out using the Statistical Package for Social Science (SPSS V.20)

\section{RESULTS}

Table 1 shows the biochemical and physicochemical properties of stabilized and un-stabilized soil samples.

Table 1. Microbiological, biochemical, and physicochemical properties of soils at the experimental site.

\begin{tabular}{lcc}
\hline Soil characteristics & Unsettled soil & Settled soil \\
\hline Soil type & Ferric luvisols & \\
Total nitrogen (\%) & 0.12 & 0.11 \\
Available phosphorus (ppm) & 12.04 & 11.51 \\
Organic matter $(\%)$ & 2.29 & 2.20 \\
& & \\
Soil electrical conductivity $(\mu \mathrm{S} / \mathrm{cm})$ & 260.00 & 258.00 \\
pH & 6.90 & 6.70 \\
Soil moisture $(\mathrm{g})$ & 19.21 & 19.00 \\
Sand $(\mathrm{g} / \mathrm{kg})$ & 640.00 & 639.00 \\
Silt $(\mathrm{g} / \mathrm{kg})$ & 160.00 & 158.00 \\
Clay $(\mathrm{g} / \mathrm{kg})$ & 169.50 & 167.00 \\
\hline
\end{tabular}

Tables 2, 4, and 6 show the identified bacteria found in the soils treated with bio-insecticides and synthetic insecticides (neem leaf extract, ginger extract, cypermethrin and chlorpyrifos insecticides, respectively). Pseudomonas aeruginosa was commonly identified in all soil samples (Table 2,
Table 4 and Table 6). Fungi identified are shown In Tables 3, 5, and 7 (neem leaf extract, ginger extract, cypermethrin, and chlorpyrifos insecticides, respectively). Aspergillus niger was the most commonly occurring fungus in all soil samples (Tables 3, 5 and 7).

Table 2. Bacteria present in soils treated with neem leaf extract.

\begin{tabular}{|c|c|}
\hline $\begin{array}{l}\text { Treatment } \\
(\mathrm{g} / \mathrm{mL})\end{array}$ & Bacteria \\
\hline Control & $\begin{array}{l}\text { Bacillus macerans, Pseudomonas chlororaphis, Pseudomonas aureginosa, Bacillus } \\
\text { lincheniformis, Proteus vulgaricus, Bacillus macquariensis, Bacillus polymyxa, Streptococcus } \\
\text { pyogenes, Micrococcus varians, Bacillus subtilis }\end{array}$ \\
\hline 100 & $\begin{array}{l}\text { Staphylococcus aureus, Pseudomonas aureginosa, Proteus mirabilis, Micrococcus luteus, } \\
\text { Bacillus macerans, Pseudomonas putida, Pseudomonas purrefaciences, Bacillus subtilis, } \\
\text { Staphylococcus aureus, Proteus vulgaricus, Serratia liquefaciens. }\end{array}$ \\
\hline 200 & $\begin{array}{l}\text { Micrococcus varians, Pseudomonas chlororaphis, Bacillus lincheniformis, Serratia marcences, } \\
\text { Streptococcus lactis, Micrococcus varians, Pseudomonas aureginosa, Bacillus subtilis, Serratia } \\
\text { liquefaciens, Proteus Mirabilis. }\end{array}$ \\
\hline 300 & $\begin{array}{l}\text { Bacillus subtilis, Pseudomonas chlororaphis, Proteus mirabilis, Staphylococcus aureus, Serratia } \\
\text { marscences, Streptococcus pyogenes, Bacillus macerans, Pseudomonas aureginosa, B. } \\
\text { macquariensis, Micrococcus varians. }\end{array}$ \\
\hline 400 & $\begin{array}{l}\text { Proteus morganii, Aeromonas hydrophilla, Bacillus subtilis, Streptococcus pyogenes, Bacillus } \\
\text { subtilis, Serratia marcences, Bacillus macerans, Bacillus subtilis, Streptococcus pyogenes, } \\
\text { Pseudomonas aeruginosa. }\end{array}$ \\
\hline 500 & $\begin{array}{l}\text { Serratia marcences, Aeromonas hydrophilla, Pseudomonas florescences, Staphylococcus } \\
\text { aureus, Bacillus macerans, Bacillus subtilis, Pseudomonas chlororaphis, Proteus mirabilis, } \\
\text { Staphylococcus aureus, Serratia marcences, Streptococcus Pyogenes. }\end{array}$ \\
\hline
\end{tabular}


Table 3. Fungi present in soils treated with neem leaf extract.

\begin{tabular}{|c|c|}
\hline $\begin{array}{l}\text { Treatment } \\
(\mathrm{g} / \mathrm{mL})\end{array}$ & Fungi \\
\hline Control & Rhizopus nigricans, Penicillum oxalicum, Aspergillus niger, Fusarium oxysporum \\
\hline $\begin{array}{l}100 \\
200\end{array}$ & $\begin{array}{l}\text { Aspergillus niger, Fusarium oxysporum, Penicillum oxalicum. } \\
\text { Aspergillus tamarii, Aspergillus fumigatus, Penicillum oxalicum, Rhizopus nigricans, Fusarium } \\
\text { compacticum, Aspergillus niger }\end{array}$ \\
\hline 300 & Aspergillus niger, Fusarium compacticum, Penicillum chrysogenum, Aspergillus fumigatus \\
\hline 400 & Aspergillus niger, Fusarium oxysporum, Aspergillus tamarii \\
\hline 500 & Penicillum chrysogenum, Fusarium oxysporum, Aspergillus niger, Aspergillus tamari \\
\hline
\end{tabular}

Table 4. Bacteria present in soils treated with ginger extract.

\begin{tabular}{|c|c|}
\hline $\begin{array}{l}\text { Treatments (g/ } \\
\mathrm{mL} \text { ) }\end{array}$ & Bacteria \\
\hline Control & $\begin{array}{l}\text { Bacillus macerans, Pseudomonas chlororaphis, Pseudomonas aureginosa, Bacillus } \\
\text { lincheniformis, Proteus vulgaricus, Bacillus macquariensis, Bacillus polymyxa, Streptococcus } \\
\text { pyogenes, Micrococcus varians, Pseudomonas chlororaphis, Bacillus subtilis. }\end{array}$ \\
\hline 100 & $\begin{array}{l}\text { Micrococcus luteus, Bacillus lincheniformis, Proteus mirabilis, Staphylococcus aureus, } \\
\text { Pseudomonas florescences, Streptococcus pyogenes, Bacillus subtilis, Micrococcus varians, } \\
\text { Bacillus lincheniformis, Streptococcus pyogenes, Pseudomonas putida, Staphylococcus } \\
\text { aureus. }\end{array}$ \\
\hline 200 & $\begin{array}{l}\text { Pseudomonas aureginosa, Micrococcus varians, Bacillus subtilis, Staphylococcus aureus, } \\
\text { Serratia marcences, Proteus mirabilis, Bacillus subtilis, Micrococcus varians, Bacillus } \\
\text { lincheniformis, Staphylococcus aureus, Pseudomonas florescences, Proteus mirabilis. }\end{array}$ \\
\hline 300 & $\begin{array}{l}\text { Bacillus subtilis, Pseudomonas aureginosa, Bacillus macerans, Pseudomonas chlororaphis, } \\
\text { Micrococcus varians, Proteus mirabilis, Bacillus macerans, Pseudomonas putida, Bacillus } \\
\text { macerans, Pseudomonas chlororaphis, Micrococcus luteus, Proteus Mirabilis. }\end{array}$ \\
\hline 400 & $\begin{array}{l}\text { Bacillus lincheniformis, Pseudomonas aureginosa, Aeromonas hydrophilla, Serratia } \\
\text { liquefaciens, Proteus mirabilis, Bacillus macerans, Pseudomonas florescences, Pseudomonas } \\
\text { chlororaphis, Micrococcus luteus, Proteus mirabilis, Bacillus subtilis. }\end{array}$ \\
\hline 500 & $\begin{array}{l}\text { Bacillus macerans, Pseudomonas florescences, Aerobacter aerogenes, Staphylococcus aureus, } \\
\text { Streptococcus pyogenes, Bacillus subtilis, Pseudomonas putida, Aerobacter aerogenes, } \\
\text { Bacillus macerans, Proteus mirabilis. }\end{array}$ \\
\hline
\end{tabular}

Table 5. Fungi present in soils treated with ginger extract.

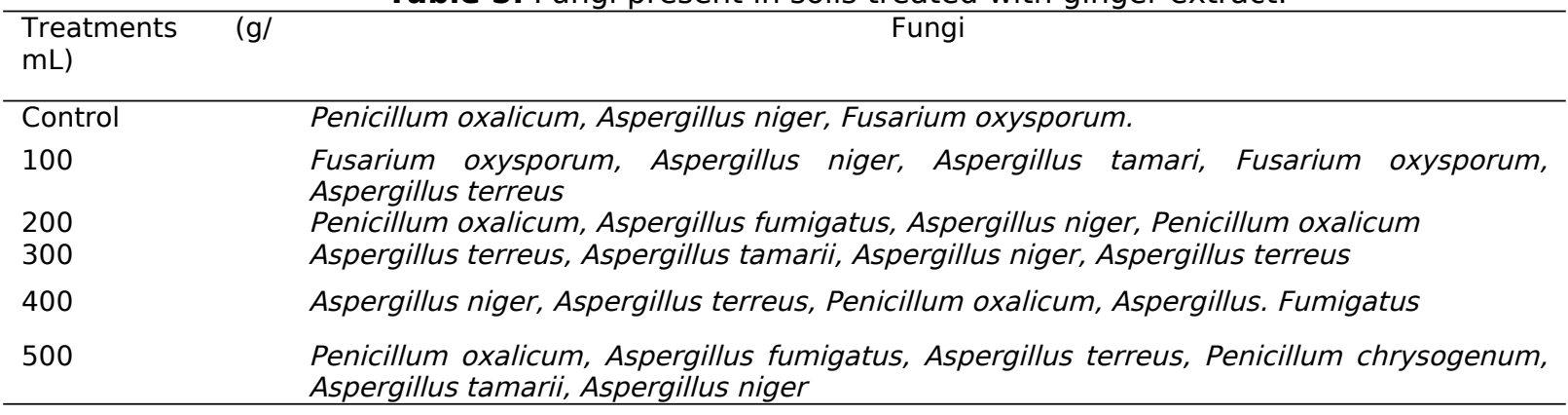

Table 6. Bacteria present in soils treated with cypermethrin and chlopyrifos.

\begin{tabular}{ll}
\hline Treatments & Bacteria \\
\hline Control & Bacillus macerans, Pseudomonas chlororaphis, Pseudomonas aureginosa, Bacillus \\
& lincheniformis, Proteus vulgaricus, Bacillus macquariensis, Bacillus polymyxa, \\
& Streptococcus pyogenes, Micrococcus varians, Pseudomonas chlororaphis, Bacillus \\
& subtilis. \\
Cypermethrin & Bacillus subtilis, Pseudomonas florescences, Aerobacter aerogenes, Bacillus \\
& lincheniformis, Serratia liquefaciens, Bacillus macerans, Pseudomonas florescences, \\
Chlorpyrifos & Aeromonas hydrophilla, Bacillus lincheniformis, Serratia marcences. \\
& Pseudomonas purrefaciences, Pseudomonas florescences, Aeromonas hydrophilla, \\
& Bacillus macerans, Bacillus subtilis, Pseudomonas aureginosa, Pseudomonas putida, \\
& Aerobacter aerogenes, Bacillus lincheniformis, Bacillus subtilis. \\
\hline
\end{tabular}


Table 7. Fungi present in soils treated with cypermethrin and chlorpyrifos.

Treatments Fungi

\begin{tabular}{ll}
\hline Control & Penicillum oxalicum, Aspergillus niger, Fusarium oxysporum \\
Cypermethrin & Penicillum oxalicum, Aspergillus niger, Aspergillus terreus, Fusarium Compacticum \\
Chlorpyrifos & Aspergillus terreus, Fusarium oxysporum, Aspergillus tamarii \\
\hline
\end{tabular}

Effects of synthetic insecticides on soil treated with chlorpyrifos recorded the lowest total microbial population coliform count of $0.10 \times 10^{5} \pm 0.00 \mathrm{cfu} / \mathrm{g}$, while The TBC, TCC and TFC of the control soil samples were significantly higher $(P \leq 0.05)$ than those of the insecticide treated soils (Table 8). Treatment with the synthetic insecticides caused significant changes $(P \leq 0.05)$ in the population of microorganisms. Control soil samples recorded the highest bacterial, total coliform and total fungal counts of $1.30 \times 10^{5} \pm 0.058 \mathrm{cfu} / \mathrm{g}, 0.73 \times 10^{5} \pm 0.048$ $\mathrm{cfu} / \mathrm{g}$ and $0.60 \times 10^{5} \pm 0.00 \mathrm{cfu} / \mathrm{g}$ respectively. Soils cypermethrin treated soils recorded the lowest total bacterial count of $0.58 \times 10^{5} \pm 0.025 \mathrm{cfu} / \mathrm{g}$. The lowest total fungal count of $0.20 \pm 0.00 \times 10^{5} \mathrm{cfu} / \mathrm{g}$ were recorded in cypermethrin and chlorpyrifos treated soils respectively. There were significant differences $(P \leq 0.05)$ in the total bacterial count, total coliform count and total fungi counts in Table 8.

Table 8. Effects of cypermethrin and chlopyrifos on soil microbial population $\left(\times 10^{5} \mathrm{cfu} / \mathrm{g}\right)$.

\begin{tabular}{llll}
\hline Treatments & TBC & TCC & TFC \\
\hline Control & $1.30 \pm 0.058^{\mathrm{c}}$ & $0.73 \pm 0.048^{\mathrm{b}}$ & $0.60 \pm 0.00^{\mathrm{b}}$ \\
Cypermethrin & $0.58 \pm 0.025^{\mathrm{a}}$ & $0.20 \pm 0.00^{\mathrm{a}}$ & $0.20 \pm 0.00^{\mathrm{a}}$ \\
Chlorpyrifos & $0.78 \pm 0.025^{\mathrm{b}}$ & $0.10 \pm 0.00^{\mathrm{a}}$ & $0.20 \pm 0.00^{\mathrm{a}}$ \\
\hline
\end{tabular}

Control = Soil with Celosia argentia but without insecticides. ANOVA showed that values were significantly different at $P<0.05$. Mean values with the same letters in a column are not significantly different $(P \geq 0.05)$, while mean values with different letters in a column are significantly different $(\mathrm{P} \leq 0.05)$.

\section{Effect of neem leaf extract on soil microbial population}

In Table 9, the soil samples treated with insecticides caused significant changes $(P \leq 0.05)$ in the microbial population. The highest TBC, TCC and TFC values of $1.30 \times 10^{5} \pm 0.058 \mathrm{cfu} / \mathrm{g}, 0.73 \times 10^{5} \pm 0.048 \mathrm{cfu} / \mathrm{g}$ and $0.60 \times 10^{5} \pm 0.00 \mathrm{cfu} / \mathrm{g}$ respectively were obtained in control soil samples. Soils treated with neem leaf extract at $500 \mathrm{~g} / \mathrm{mL}$ recorded the lowest total bacterial, total fungal, and total coliform counts of $0.38 \pm 0.025 \times 10^{5} \mathrm{cfu} / \mathrm{g}, 0.20 \pm 0.00 \times 10^{5} \mathrm{cfu} / \mathrm{g}$ and $0.20 \times 10^{5} \pm 0.00 \mathrm{cfu} / \mathrm{g}$, respectively.

Table 9. Effects of neem leaf extracts on soil microbial population $\left(\times 10^{5} \mathrm{cfu} / \mathrm{g}\right)$.

\begin{tabular}{llll}
\hline $\begin{array}{l}\text { Treatments } \\
(\mathrm{g} / \mathrm{ml})\end{array}$ & TBC & TCC & TFC \\
\hline Control & $1.30 \pm 0.058^{\mathrm{f}}$ & $0.73 \pm 0.048^{\mathrm{c}}$ & $0.60 \pm 0.00^{\mathrm{d}}$ \\
100 & $1.15 \pm 0.29^{\mathrm{e}}$ & $0.68 \pm 0.025^{\mathrm{c}}$ & $0.50 \pm 0.00^{\mathrm{c}}$ \\
200 & $0.93 \pm 0.48^{\mathrm{d}}$ & $0.53 \pm 0.025^{\mathrm{b}}$ & $0.35 \pm 0.029^{\mathrm{b}}$ \\
300 & $0.70 \pm 0.00^{\mathrm{c}}$ & $0.45 \pm 0.029^{\mathrm{b}}$ & $0.45 \pm 0.029^{\mathrm{c}}$ \\
400 & $0.55 \pm 0.029^{\mathrm{b}}$ & $0.30 \pm 0.00^{\mathrm{a}}$ & $0.28 \pm 0.025^{\mathrm{a}}$ \\
500 & $0.38 \pm 0.025^{\mathrm{a}}$ & $0.20 \pm 0.00^{\mathrm{a}}$ & $0.20 \pm 0.00^{\mathrm{a}}$ \\
\hline
\end{tabular}

Control $=$ Soil with Celosia argentia but without insecticides. ANOVA showed that values were significantly different at $P<0.05$. Mean values with the same letters in a column are not significantly different $(P \geq 0.05)$, while mean values with different letters in a column are significantly different $(P \leq 0.05)$.

\section{Effect of ginger extract on soil microbial} population

In Table 10, the control soil samples recorded the highest TBC, TCC and TFC values. Soil treated with ginger extract at $500 \mathrm{~g} / \mathrm{mL}$ recorded the lowest TBC,
TCC and TFC values of $0.30 \pm 0.00 \times 10^{5} \mathrm{cfu} / \mathrm{g}$ and $0.20 \pm 0.00 \times 10^{5} \mathrm{cfu}$ respectively. There were significant differences $(P \leq 0.05)$ in the total bacterial count, total coliform count and total fungal count at different concentrations. 
Table 10. Effects of ginger extracts on soil microbial population $\left(\times 10^{5} \mathrm{cfu} / \mathrm{g}\right)$

\begin{tabular}{llll}
\hline $\begin{array}{l}\text { Treatments }(\mathrm{g} / \\
\mathrm{L})\end{array}$ & TBC & TCC & TFC \\
\hline Control & $1.30 \pm 0.058^{\mathrm{d}}$ & $0.73 \pm 0.048^{\mathrm{c}}$ & $0.60 \pm 0.00^{\mathrm{d}}$ \\
100 & $1.23 \pm 0.025^{\mathrm{d}}$ & $0.78 \pm 0.025^{\mathrm{c}}$ & $0.43 \pm 0.25^{\mathrm{c}}$ \\
200 & $0.73 \pm 0.048^{\mathrm{c}}$ & $0.58 \pm 0.025^{\mathrm{b}}$ & $0.40 \pm 0.00^{\mathrm{c}}$ \\
300 & $0.48 \pm 0.025^{\mathrm{b}}$ & $0.30 \pm 0.00^{\mathrm{a}}$ & $0.30 \pm 0.00^{\mathrm{b}}$ \\
400 & $0.45 \pm 0.029^{\mathrm{b}}$ & $0.25 \pm 0.29^{\mathrm{a}}$ & $0.20 \pm 0.00^{\mathrm{a}}$ \\
500 & $0.30 \pm 0.00^{\mathrm{a}}$ & $0.20 \pm 0.00^{\mathrm{a}}$ & $0.20 \pm 0.00^{\mathrm{a}}$ \\
\hline
\end{tabular}

Control= Soil with Celosia argentia but without insecticides. ANOVA showed that values were significantly different at $P<0.05$. Mean values with the same letters in a column are not significantly different $(P \geq 0.05)$, while mean values with different letters in a column are significantly different $(\mathrm{P} \leq 0.05)$.

\section{Effects of insecticides on microbial activities}

Table 11 shows the effect of insecticides on dehydrogenase activity in the soils. Treatment of soil samples with the synthetic and biological insecticides resulted in significant drop in dehydrogenase activity. The control soil sample had the highest dehydrogenase activity value of $30.35 \pm$
$0.050 \mu \mathrm{g} \mathrm{g}^{-1} \mathrm{~h}^{-1}$, followed by $500 \mathrm{~g} / \mathrm{mL}$ Neem leaf extract $\left(27.95 \pm 0.250 \mu \mathrm{g} \mathrm{g} \mathrm{g}^{-1} \mathrm{~h}^{-1}\right)$. Chlorpyrifos treated soils had the lowest dehydrogenase activity value of $17.50 \pm 0.100 \mu \mathrm{g} \mathrm{g}^{-1} \mathrm{~h}^{-1}$. There were significant changes $(P \leq 0.05)$ in the values of dehydrogenase activities in the insecticide treated soils.

Table 11. Effects of insecticides on dehydrogenase activities in the soil.

\begin{tabular}{|c|c|c|}
\hline 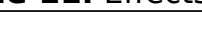 & Concentration (g/L) & $\operatorname{DEH}\left(\mu \mathrm{g} \mathrm{g}^{-1} \mathrm{~h}^{-1}\right)$ \\
\hline Control & & $30.35 \pm 0.050^{1}$ \\
\hline \multirow[t]{5}{*}{ Neem leaf } & 100 & $21.95 \pm 0.150^{\text {de }}$ \\
\hline & 200 & $21.95 \pm 0.150^{\mathrm{de}}$ \\
\hline & 300 & $22.70 \pm 0.100^{f}$ \\
\hline & 400 & $27.60 \pm 0.100^{\mathrm{h}}$ \\
\hline & 500 & $27.95 \pm 0.250^{h}$ \\
\hline \multirow[t]{6}{*}{ Ginger } & 100 & $21.55 \pm 0.050^{\text {cd }}$ \\
\hline & 200 & $21.25 \pm 0.150^{c}$ \\
\hline & 300 & $22.20 \pm 0.100^{c}$ \\
\hline & 400 & $26.35 \pm 0.150^{g}$ \\
\hline & 500 & $26.70 \pm 0.100^{g}$ \\
\hline & Concentration $(\mathrm{mL})$ & DEH $\left(\mu \mathrm{g} \mathrm{g}^{-1} \mathrm{~h}^{-1}\right)$ \\
\hline Cypermethrin & 13 & $18.35 \pm 0.150^{b}$ \\
\hline Chlorpyrifos & 13 & $17.50 \pm 0.100^{\mathrm{a}}$ \\
\hline
\end{tabular}

ANOVA showed that values were significantly different at $\mathrm{P}<0.05$. Mean values with the same letters in a column are not significantly different $(P \geq 0.05)$, while mean values with different letters in a column are significantly different $(P \leq 0.05)$.

In Table 12, microbial biomass carbon of soil samples reduced significantly after treatment with the insecticides. It was observed that $500 \mathrm{~g} / \mathrm{mL}$ Neem leaf had the highest microbial biomass carbon value of $9.40 \pm 0.100 \mathrm{~kg} \mathrm{C} / \mathrm{m}^{2}$, followed by $400 \mathrm{~g}$
Neem leaf $\left(8.40 \pm 0.200 \mathrm{~kg} \mathrm{C} / \mathrm{m}^{2}\right)$. Control soil samples recorded microbial biomass carbon value of $5.80 \pm 0.100 \mathrm{~kg} \mathrm{C} / \mathrm{m}^{2}$. Meanwhile, cypermethrin recorded the lowest microbial biomass carbon value of $2.05 \pm 0.250 \mathrm{~kg} \mathrm{C} / \mathrm{m}^{2}$. 
Table 12. Effects of insecticides on microbial biomass carbon $\left(\mathrm{kg} \mathrm{C} / \mathrm{m}^{2}\right)$ in soil samples.

\begin{tabular}{|c|c|c|}
\hline & Concentration(g/L) & $\mathrm{MBC}\left(\mathrm{kg} \mathrm{C} / \mathrm{m}^{2}\right)$ \\
\hline Control & & $5.80 \pm 0.100^{\mathrm{e}}$ \\
\hline \multirow[t]{5}{*}{ Neem leaf } & 100 & $5.35 \pm 0.150^{\mathrm{de}}$ \\
\hline & 200 & $6.70 \pm 0.200^{1}$ \\
\hline & 300 & $8.05 \pm 0.250^{\mathrm{hi}}$ \\
\hline & 400 & $8.40 \pm 0.200^{i}$ \\
\hline & 500 & $9.40 \pm 0.100^{J}$ \\
\hline \multirow[t]{6}{*}{ Ginger } & 100 & $4.00 \pm 0.100^{h}$ \\
\hline & 200 & $4.55 \pm 0.150^{\mathrm{bc}}$ \\
\hline & 300 & $5.05 \pm 0.150^{c d}$ \\
\hline & 400 & $7.45 \pm 0.150^{g}$ \\
\hline & 500 & $7.65 \pm 0.150^{g h}$ \\
\hline & Concentration $(\mathrm{mL})$ & $\mathrm{MBC}\left(\mathrm{KgC} / \mathrm{m}^{2}\right)$ \\
\hline Cypermethrin & 13 & $2.05 \pm 0.250^{\mathrm{a}}$ \\
\hline Chlorpyrifos & 13 & $2.50 \pm 0.300^{\mathrm{a}}$ \\
\hline
\end{tabular}

ANOVA showed that values were significantly different at $P<0.05$. Mean values with the same letters in a column are not significantly different $(P \geq 0.05)$, while mean values with different letters in a column are significantly different $(P \leq 0.05)$.

Table 13 shows the effects of insecticides on microbial respiration $\left(\mathrm{CO}_{2}\right.$ respired). The insecticides also caused significant reduction in microbial respiration after treatment with the insecticides. 500 $\mathrm{g} / \mathrm{mL}$ of Neem leaf extract had the highest microbial respiration value of $2288.00 \pm 1.000 \mathrm{mg} / \mathrm{kg}$. The control soil samples recorded a value of $1915.50 \pm$ $1.500 \mathrm{mg} / \mathrm{kg}$. $100 \mathrm{~g}$ of Neem leaf and $200 \mathrm{~g}$ of Ginger were observed to have a similar value of $1872.50 \pm 1.500 \mathrm{mg} / \mathrm{kg}$. Cypermethrin treated soils had the lowest effect on $\mathrm{CO}_{2}$ respired in the soil with a value of $1687.50 \pm 1.500 \mathrm{mg} / \mathrm{kg}$. There were significant differences $(P \leq 0.05)$ in the microbial respiration values of the insecticide treated soils.

\section{DISCUSSION AND CONCLUSION}

In this study, Pseudomonas aeruginosa and Aspergillus niger were the most commonly isolated microorganisms from the insecticide-treated soil samples. Iqbal and Bartakke (10) reported that they isolated Acinetobacter radioresistens, Pseudomonas frederiksbergensis, Bacillus pumilus, Serratia liquefaciens, Serratia marcescens, and Burkholderia gladioli. Treatment of soil samples with neem leaf extracts resulted in significant reduction in soil microbes. This is supported by the finding of Nasim et al. (11) who also confirmed the same result for neem extracts. Neem extracts have been reported to possess antibacterial, antifungal, antimalarial, and antiviral properties.

Ajaz et al. (12) reported the isolation of chlorpyrifos resistant bacteria from cotton cultivated soil using conventional and API kit methods (12). In the present study, reduction in bacterial populations was observed after field treatment with chlorpyrifos. Liu et al. (13) reported reduced microbial populations which may affect the quantity of organic matter in soils. The extensive use of chlorpyrifos having a half-life from 10 to 120 days in soil has resulted in widespread environmental contamination affecting beneficial non-target soil microorganisms. Bera et al. (14) stated that microorganisms can cause breakdown of insecticides and utilize them as a source of nutrients. However, before degradation, insecticides have toxic effects on microorganisms, reducing their abundance, activity, and consequently, the diversity of their communities. 
Table 13. Effects of insecticides on microbial respiration $\left(\mathrm{CO}_{2}\right.$ respired) in the soil.

\begin{tabular}{|c|c|c|}
\hline & $\begin{array}{l}\text { Concentration } \\
(\mathrm{g} / \mathrm{mL})\end{array}$ & $\mathrm{CO}_{2}$ respired $(\mathrm{mg} / \mathrm{kg})$ \\
\hline Control & & $1915.50 \pm 1.500^{f}$ \\
\hline \multirow[t]{5}{*}{ Neem leaf } & 100 & $1872.50 \pm 1.500^{d}$ \\
\hline & 200 & $1877.00 \pm 1.000^{\mathrm{de}}$ \\
\hline & 300 & $1880.50 \pm 1.500^{\mathrm{e}}$ \\
\hline & 400 & $2184.50 \pm 1.500^{h}$ \\
\hline & 500 & $2288.00 \pm 1.000^{\mathrm{i}}$ \\
\hline \multirow[t]{6}{*}{ Ginger } & 100 & $1867.50 \pm 1.500^{\circ}$ \\
\hline & 200 & $1872.50 \pm 1.500^{\circ}$ \\
\hline & 300 & $1865.50 \pm 1.500^{\circ}$ \\
\hline & 400 & $1970.00 \pm 2.000^{\mathrm{g}}$ \\
\hline & 500 & $1974.00 \pm 1.000^{\mathrm{g}}$ \\
\hline & $\begin{array}{l}\text { Concentration } \\
(\mathrm{mL})\end{array}$ & $\mathrm{CO}_{2}$ respired $(\mathrm{mg} / \mathrm{kg})$ \\
\hline Cypermethrin & 13 & $1687.50 \pm 1.500^{\mathrm{a}}$ \\
\hline BTermicot & 13 & $1694.00 \pm 2.000^{b}$ \\
\hline
\end{tabular}

ANOVA showed that values were significantly different at $\mathrm{P}<0.05$. Mean values with the same letters in a column are not significantly different $(P \geq 0.05)$, while mean values with different letters in a column are significantly different $(P \leq 0.05)$.

The toxic effects of insecticides are initially noticed in soils by the reduction of microbial enzymic activities and microbial populations after application. After adaptation of microorganisms to these toxic environmental conditions, there is an increase in microbial enzymic activities and microbial population which in turn results in degradation of these toxic insecticides. In this study, the effects of insecticides on soil microbial enzymes (dehydrogenase and microbial respiration) showed reduction in microbial activity after treatment with the insecticides (synthetic and biological insecticide), the observed trends in relation to microbial enzymes in this research work were similar to the one we initially made (15). Rasool and Reshi (16) reported that several insecticides suppressed and reduced the activity of dehydrogenase enzymes in their study.

Caceres et al. (17) stated that pesticides have negative impacts of pesticides on soil enzymes such as hydrolases, oxidoreductases and dehydrogenase activities. Gundi (18) determined the effect of monochrotophos, quinalphos and cypermethrin on microbial populations in a black clay soil and demonstrated their synergistic effects at the lower levels and adverse effects at the highest level of the insecticides. According to Lopez (19) heterotrophic mesophilic and psychrophilic aquatic bacteria as well as culturable phosphate-solubilizing microorganisms increased in lake water samples when treated with insecticides showing that sometimes, initially microbial population is affected by insecticide application but with time after adaptation to these insecticides, the population merely returns to normal or even increases. This indicates the changes in microbial catabolic capabilities as a result of induced insecticide degradation capabilities or due to a change within the microbial community.

The synthetic insecticides used in this study showed higher negative impacts on microbial activities (microbial biomass carbon, microbial respiration, dehydrogenase activity and microbial population) compared to the biological insecticides which were applied at different concentrations.

\section{CONCLUSION}

This work has shown that treatment with both synthetic and bio-insecticides resulted in significant drop in microbial population and microbial activity of soil samples. The synthetic insecticides used in this study has been shown to cause major reduction in microbial population and microbial activity. Hence 
the use of synthetic insecticides is discouraged. Further research could be carried out to improve the usability of the biological insecticides.

\section{REFERENCES}

1. Zhang B, Bai Z, Hoefel D, Tang L, Wang X, Li B, Li Z, Zhuang G. The impacts of cypermethrin pesticide application on the non-target microbial community of the pepper plant phyllosphere. Science on Total Environment. 2009; 407:1915-22.

2. Ramudu A, Mohiddin G, Srinivasulu $M$, Rangaswamy $V$. Impact of fungicides chlorothaln oil and propiconazole on microbial activities in groundnut (Arachis hypogaea L.) soils. International Scholarly Research Network Microbiology. 2011; 623404, 1-7.

3. Nannipieri P, Cervelli S, Pema A. Enzyme activities in some Italian soils. Journal of Italian Agriculture. 2012; 73: 367-76.

4. Diplock EE, Mardlin DP, Killham KS, Paton G I. Predicting bioremediation of hydrocarbons: laboratory to field scale. Environmental Pollution. 2009; 157 (6): 1831-40.

5. Das AC, Mukherjee D. Soil application of insecticides influences microorganisms and Plant Nutrient Applied Soil Ecology. 2007;14: 55-62.

6. Vig K, Singh DK, Agrarwal HC, Dhawan AK, Dureja $P$. Soil micro- organisms in cotton fields sequencially treated with insecticides. Ecotoxicology and Environmental Safety. 2009; 69:263-76.

7. Sarfraz H, Siddique T, Saleem M, Arshad M, Khalid A. Impact of pesticides on soil microbial diversity, enzymes and biochemical reactions. Advances in Agronomy. 2009; 102:159-200.

8. Klimek B. Effect of Long-Term Zinc Pollution on Soil Microbial Community Resistance to Repeated Contamination. Bulletin of Environmental Contamination and Toxicology. 2012; 8(4): 617-22.

9. Vance ED, Brookes PC, Jenkinson DS. An extraction method for measuring soil microbial biomass C. Soil Biology and Biochemistry. 1987; 17 (6): $837-42$.
10. Iqbal MA, Bartakke KV. Isolation of Pesticide Degrading Microorganisms from soil. Advances in Bioresearch. 2014; 5(4):164-8.

11. Nasim G, Ilyas N, Shabbir A. Study of effect of organic pesticides: Endosulfan and Bifenthrin on growth of some soil fungi. Mycopathology. 2005; 3 (1\&2): 27-31.

12. Ajaz M, Jabeen N, Akhtar S, Rasool SA.

Cloropyrifos resistant bacteria from Pakistani soils: isolation, identification, resistance profile and growth profile. Pakistan Journal of Botany. 2005; 37(2): 381-8.

13. Liu J, Xie J, Chu Y, Sun C, Chen C, Wang Q. Combined effect of cypermethrin and copper on catalase activity in soil. J. Soils Sed. 2008; 8: 327-32.

14. Bera S, Ghosh RK. Soil Microflora and Weed Management as Influenced byAtrazine 50 \% WP in Sugarcane. Universal Journal of Agricultural Research. 2013; 1(2): 41-7.

15. Sebiomo A, Ogundero VW, Bankole SA. Effect of four herbicides onmierobial population, soil organic matter and dehydrogenase activity. African Journal of Biology. 2012; 10(5): 770-8.

16. Rasool N, Reshi, ZA. Effect of the fungicide Mancozeb at different application rates on enzyme activities in a silt loam soil of the Kashmir Himalaya, India, Trop. Ecol. 2010; 51; 199-205.

17. Caceres TP, He WX, Megharaj M, Naidu, R. Effect of insecticide fenamiphos on soil microbial activities in Australian and Ecuadorean soils. Journal of Environmental Science. 2009; 44: 13-7.

18. Gundi V, Viswanath B, Chandra MS, Kumar VN, Reddy BR. Activities of cellulase and amylase in soils as influenced by insecticide interactions. Ecotoxicology and Environmental Safety. 2007; 68: 278-85.

19. Lopez J. Growth and denitrifying activity of Xanthobacter in the presence of selected pesticides. Applied Microbiology and Biotechnology. 2006; 71: 563-7.

20. Min H, Ye YF, Chen ZY, Wu WX, Du YF. Effects of butachlor on microbial populations and enzyme activities in paddy soil. Journal of Environmental Science and Health. 2001; 36: 581-95. 
